\title{
Smart textile for respiratory monitoring and thoraco-abdominal motion pattern evaluation
}

\author{
Carlo Massaroni $^{1 *}$ | Cecilia Venanzi ${ }^{1}$ | Amanda P. Silvatti ${ }^{2}$ | Daniela Lo Presti ${ }^{1}$ | \\ Paola Saccomandi $^{3}$ | Domenico Formica ${ }^{4}$ | Francesco Giurazza ${ }^{1}$ | Michele A. Caponero ${ }^{5}$ | \\ Emiliano Schena ${ }^{1}$
}

${ }^{1}$ Unit of Measurements and Biomedical Instrumentation, Department of Engineering, Università Campus Bio-Medico di Roma, Rome, Italy

${ }^{2}$ Department of Physical Education, Universidade Federal de Viçosa Minas Gerais, Brazil

${ }^{3}$ Institute of Image-Guided Surgery, Strasbourg, France

${ }^{4}$ Unit of Neurophysiology and Neuroengineering of Human-Technology Interaction, Università

Campus Bio-Medico di Roma, Rome, Italy

${ }^{5}$ Photonics Micro- and Nanostructures Laboratory, Research Centre of Frascati, Rome, Italy

*Correspondence

Carlo Massaroni, Unit of Measurements and Biomedical Instrumentation, Department of Engineering, Università Campus Bio-Medico di Roma, via Álvaro del Portillo, 21, 00128 Rome, Italy.

Email: c.massaroni@unicampus.it
The use of wearable systems for monitoring vital parameters has gained wide popularity in several medical fields. The focus of the present study is the experimental assessment of a smart textile based on 12 fiber Bragg grating sensors for breathing monitoring and thoraco-abdominal motion

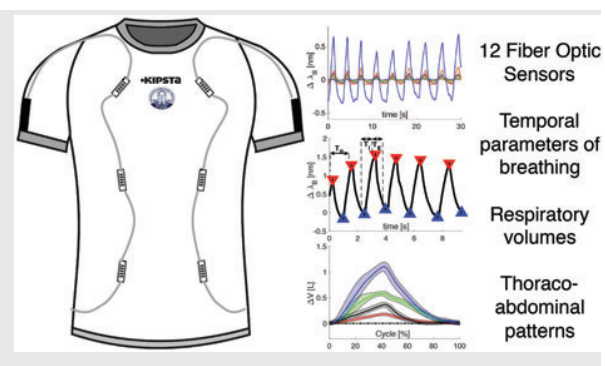
pattern analysis. The feasibility of the smart textile for monitoring several temporal respiratory parameters (ie, breath-by-breath respiratory period, breathing frequency, duration of inspiratory and expiratory phases), volume variations of the whole chest wall and of its compartments is performed on 8 healthy male volunteers. Values gathered by the textile are compared to the data obtained by a motion analysis system, used as the reference instrument. Good agreement between the 2 systems on both respiratory period (bias of 0.01 seconds), breathing frequency (bias of -0.02 breaths $/ \mathrm{min}$ ) and tidal volume (bias of $0.09 \mathrm{~L}$ ) values is demonstrated. Smart textile shows good performance in the monitoring of thoraco-abdominal pattern and its variation, as well.

\section{KE YWORDS}

breathing monitoring, fiber Bragg gratings, fiber optics, smart textiles, wearables

\section{1 | INTRODUCTION}

Monitoring of vital signs such as heart rate, respiratory rate, blood pressure and temperature, is relevant to assess subject's health state. Among these, respiratory parameters play an important role since their continuous monitoring may be crucial for detecting respiratory disorders such as asthma, sleep apnea, thoraco-abdominal asynchronies [1].

In this context, comfortable and easy-to-wear systems have gained popularity since they allow the continuous respiratory monitoring without limiting the subject's mobility $[2,3]$. Several technological solutions (ie, inductive, piezo-electric, capacitive, piezo-resistive sensors) have been adopted to register ventilation-induced thoracic and/or abdominal strain through sensors embedded into straps or clothes since the lung displacement results in deformations of the thoraco-abdominal surface [2].

Recently, smart textiles based on fiber optic sensors have shown promising results in the respiratory monitoring $[4,5]$. Among others, fiber Bragg grating sensors (FBG) have been profitably used in the monitoring of breathing rate by integrating sensing element in patches $[6,7]$ or garments $[8,9]$. By taking the advantage of their small size, the possibility of integrating more sensors in 1 fiber to carry 
out distributed measurements, and their high sensitivity to strain, more FBGs may be used to design comfortable wearable textiles for the analysis of local chest wall strains. The deformations of thorax and abdomen can indirectly provide an estimation of the contribution of the thoracic and abdominal compartments on the volume pattern during the breathing [10], allowing the biomechanical analysis of the chest wall. In fact, from a physiological point of view, the chest wall can be considered as a 6-compartmental structure, composed by right and left sides of the pulmonary rib cage, the abdominal rib cage, and the abdomen that reflect anatomical and muscular districts working during the breathing activity [11]. Motion capture (MoCap) systems have been extensively used on patients [12,13] and athletes [14] to investigate how the breathing biomechanics is influenced by postures, activities, diseases. These systems require the positioning of photo-reflective markers attached on the skin [15], the adoption of dedicated computational methods together with high-speed infra-red (IR) cameras. However, the high costs of cameras, the need of dedicated equipped room, professional staff required for pre- and postprocessing phase, strongly limit the use of MoCap systems especially for prolonged monitoring [13].

Our research group has been already investigated solutions based on 2 [8] and 6 [9] FBGs embedded into garments for the evaluation of both temporal and volumetric respiratory parameters of human subjects. Additionally, a promising 12 sensor-based smart textile has been designed and preliminary tested for the monitoring of average respiratory rate [16].

In this work, we investigated the feasibility of the 12 FBG-based smart textile for (i) the breath-by-breath monitoring of several breathing parameters such as breathing frequency, expiratory and inspiratory periods; (ii) the monitoring of tidal volumes and thoraco-abdominal contributions to the total volume, and their variabilities during the trials after a calibration procedure. The assessment was performed on male healthy volunteers during their self-paced breathing in standing posture. A MoCap system with passive markers was used as a gold standard instrument.

\section{I MATERIALS AND METHODS}

\section{1 | Smart textile: design and working principle}

The smart textile used in this work is a garment based on 12 FBGs we already developed [16]. The 12 sensing elements have been positioned in specific body landmarks in order to optimize the smart textile sensitivity to strain caused by the breathing activity (by lungs, diaphragm, accessory muscles) in each chest wall compartments [16].

FBG is a series of localized changes in the refractive index $\left(\eta_{\text {eff }}\right)$ of the fiber that creates the grating effect. The optical fiber is connected to a light source, which typically emits light in a broad wavelength band. When the light encounters the FBG, a narrow spectrum centred around a specific wavelength, named Bragg wavelength $\left(\lambda_{\mathrm{B}}\right)$, is reflected, while the rest of the light in the transmission band passes through the grating. The $\lambda_{\mathrm{B}}$ value depends on the properties of the gratings.

The FBG responds to both strain $(\varepsilon)$ and temperature $(T)$ [17]. A fiber strain effects the grating spatial period $(\Lambda)$ and causes a change of $\eta_{\text {eff }}$ associated with Poisson's effect (photoelastic effect) due to dimensional changes in the radial direction as in Equation (1):

$$
\lambda_{B}(\varepsilon, T)=2 \cdot \Lambda(\varepsilon, T) \cdot \eta_{e f f}(\varepsilon, T) .
$$

The change in wavelength $\Delta \lambda_{\mathrm{B}}$ associated with both $\varepsilon$ and $T$ can be expressed as Equation (2):

$\Delta \lambda_{B}(\varepsilon, T)=2 \eta_{e f f} \Lambda\left\{\frac{\eta_{e f f}^{2}}{2}\left[P_{12} \nu\left(P_{11}+P_{12}\right)\right]\right\} \varepsilon+\left(\alpha+\frac{\frac{d \eta_{e f f}}{d T}}{\eta_{e f f}}\right) \Delta T$,

where $P_{11}, P_{12}$ are the stress optic coefficients, $\alpha$ is the coefficient of thermal expansion, $v$ is the Poisson's ratio. In specific applications, where $T$ changes may be considered negligible, the $\Delta \lambda_{\mathrm{B}}$ can be linearly related to strain.

We choose to use FBGs primarily because they are well suited for distributed sensing, which implies that multiple sensing points can be distributed along a single fiber, because they are intrinsically safe (no electrical power necessary), small sized and lightweight. In addition, they can be integrated into composite or fixed directly on the surface of the object to monitor without affecting performances.

We designed the smart textile considering that the breathing activity causes significant thoraco-abdominal movements [16]. Therefore, FBG sensors glued on the textile are strained and their output shift can be related to breathing activity. The positions of the sensing elements on the textile were defined after a biomechanical analysis of the chest wall movements during quiet breathing and exercise on healthy subjects, as previously described in Reference [16]. The 12 FBG sensors (10 mm of grating length) were glued on a commercial slim fit size-M T-shirt (OXYLANE, 97\% polyamid, 3\% elastane, Kipsta, Villeneuve-d'Ascq, France) by using adhesive silicon rubber containing trimethoxy(methyl)silano (3145 RTV MILA-46146, DOW CORNING, Michigan, Michigan).

The 12 FBGs allow the local strain analysis of the 6 compartments in which the chest wall can be functionally split (the left and the right sides of the pulmonary rib cage- $\mathrm{RCp}-$, the abdominal rib cage- $\mathrm{RCa}-$ and the abdomen-AB-). Two FBGs are used to monitor each compartment according to the scheme of Figure 1.

The 12 FBG outputs are collected by a 4-channel optical spectrum interrogator (Si425 Reading Unit, Micron Optics Inc., Atlanta, USA), at a sampling rate of $250 \mathrm{~Hz}$. A dedicated Virtual Instrument in LabVIEW environment (National 
Instruments, Austin, Texas) running on a notebook provides the visualization and saving of data.

\section{2 | MoCap system}

A MoCap system was used as a gold standard instrument for estimating the following breathing parameters: breathing frequency, total duration of the breathing, expiratory and inspiratory periods, total volume changes and thoracoabdominal contributions. Eighty-nine infraredphotoreflective markers were placed on the smart textile worn by the volunteer by following the protocol described in Reference [15], using hypoallergenic tape, 42 placed on the anterior face of the chest, 37 on the posterior one and 5 additional markers per side on the lateral faces of the trunk [15, 18]. The markers' positioning is designed to allow an adequate sampling of the complex shape of the thoraco-abdominal surface and an adequate subdivision of total chest wall volume into before-mentioned 6 different compartments.

The trajectories of markers were collected by a 8-camera MoCap system (Smart-D, BTS Bioengineering Corp., Milan, Italy) at a sampling rate of $60 \mathrm{~Hz}$, and a dedicated software (ie, OEP-Smart, BTS Bioengineering Corp., Milan, Italy) was used to compute the volumes [19].

From the marker's trajectories, the volumes enclosed into the thoraco-abdominal surface were obtained through the connection of markers to constitute a net of triangles. For each triangle obtained, the area $\left(S_{i}\right)$ and direction of the normal vector $\vec{n}_{i}$ are determined. Thus, the internal volume of each shape is calculated using Gauss's theorem-in which the surface integral is converted into the volume integral-as in Equation (3):

$$
\int_{S} \vec{F}_{i} \cdot \vec{n}_{i} d S_{i}=\int_{V} d V_{i}=V_{i}
$$

being $\vec{F}_{i}$ an arbitrary vector. This procedure allows computing the volume enclosed by the 6 compartments of the thoraco-abdominal surface (left and right sides of
$\left.\Delta V_{\mathrm{RCp}}{ }^{\text {MoCap }}, \Delta V_{\mathrm{RCa}}{ }^{\text {MoCap }}, \Delta V_{\mathrm{AB}}^{\text {MoCap }}\right)$ [15]. The volume enclosed by the whole chest wall ( $\Delta V_{\text {global }}$ MoCap $)$ is mathematically obtained by the sum of these 6 compartmental volumes.

\section{3 | PROTOCOL AND EXPERIMENTAL SETUP}

Experiments were carried out to simultaneously collect the $\Delta \lambda_{\mathrm{B}}$ of all the 12 FBGs embedded into the smart textile and the 6 compartmental volumes through the MoCap system.

The following parameters obtained by the smart textile and the MoCap were compared: breath-by-breath respiratory period $\left(T_{\mathrm{R}}\right)$ and breathing frequency $\left(f_{R}\right)$, duration of inspiratory $\left(T_{\mathrm{I}}\right)$ and expiratory phases $\left(T_{\mathrm{E}}\right)$, tidal volumes (VT) extracted by global volume pattern $\left(\Delta V_{\text {global }}\right)$ and compartmental volumes changes (ie, $\Delta V_{\mathrm{RCp}}, \Delta V_{\mathrm{RCa}}, \Delta V_{\mathrm{AB}}$ ).

Eight healthy male volunteers with no history of respiratory disorders were enrolled in this study. Their anthropometric measurements and descriptive statistics are reported in Table 1. Approval for the study was obtained from the Institutional Review Board of the Universidade Federal de Viçosa, Prot. 56335515.8.0000.5153. All subjects provided their informed written consent, and the study was conducted in accordance with the latest release of the Declaration of Helsinki.

Each volunteer was invited to wear the smart textile and to assume a standing posture. Then the 89 IRphotoreflective markers were placed on the textile for the MoCap data collection. Each volunteer was instructed to perform 2 different trials of self-paced quiet breathing lasting 60 seconds each, after a brief forced inspiration used to synchronize the signals of smart textile and MoCap in the post-processing phase.

\section{4 | DATA ANALYSIS}

Data collected in the first trial by the MoCap and by the smart textile were used:

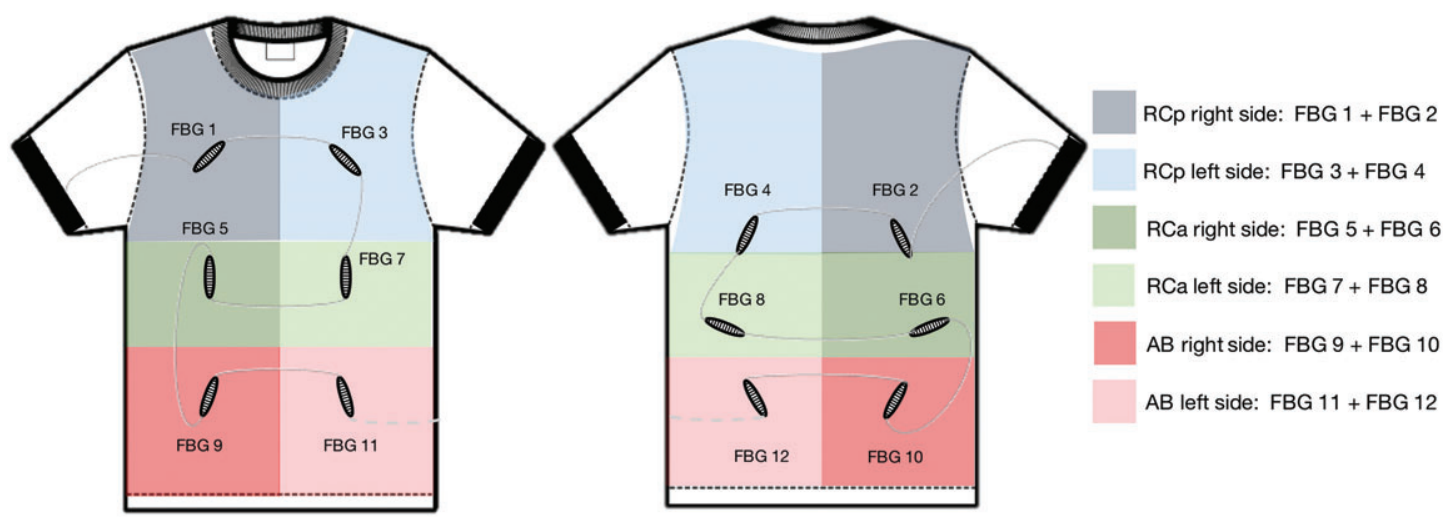

FIGURE 1 The 12 FBG sensors on the textile together with the 6 compartments configuration of the smart textile: FBG couples used to monitor each compartment are reported and explained in the text 
TABLE 1 Anthropometric data and descriptive statistics of each volunteer

\begin{tabular}{|c|c|c|c|c|c|c|c|c|}
\hline \multirow[b]{2}{*}{ Volunteer } & \multicolumn{3}{|c|}{ Descriptive statistics } & \multicolumn{5}{|c|}{ Anthropometric measurements } \\
\hline & Age (years) & Height (cm) & Weight (kg) & I line (cm) & II line (cm) & III line (cm) & IV line $(\mathrm{cm})$ & $\mathrm{V}$ line $(\mathrm{cm})$ \\
\hline 1 & 27 & 168 & 60 & 32 & 50 & 89 & 87 & 77 \\
\hline 2 & 25 & 177 & 70 & 36 & 57 & 95 & 89 & 85 \\
\hline 3 & 22 & 180 & 70 & 35 & 57 & 93 & 88 & 86 \\
\hline 4 & 23 & 172 & 67 & 37 & 53 & 97 & 94 & 85 \\
\hline 5 & 22 & 170 & 68 & 36 & 50 & 93 & 85 & 83 \\
\hline 6 & 28 & 165 & 61 & 35 & 51 & 90 & 86 & 77 \\
\hline 7 & 23 & 178 & 83 & 36 & 52 & 98 & 91 & 84 \\
\hline 8 & 22 & 174 & 72 & 35 & 51 & 96 & 90 & 86 \\
\hline $\mathrm{Avg} \pm \mathrm{SD}$ & $24 \pm 2$ & $173 \pm 5$ & $69 \pm 7$ & $35 \pm 2$ & $53 \pm 3$ & $94 \pm 3$ & $89 \pm 3$ & $83 \pm 4$ \\
\hline
\end{tabular}

Average (Avg) and standard deviation (SD) are also shown. I line: inter-acromial distance; II line: c7-coccyx distance; III line: circumference at the height of axilla; IV line: circumference at the height of xiphoid; V line: circumference at the level of anterior superior iliac spine.

i. to calculate the breathing temporal parameters $\left(T_{\mathrm{R}}, f_{R}\right.$, $\left.T_{\mathrm{I}}, T_{\mathrm{E}}\right)$ from the $\Delta V_{\text {global }}$ MoCap and the $\Delta \lambda_{\mathrm{B}}$ global and to compare the 2 systems. The values of $\Delta \lambda_{\mathrm{B}}$ global were obtained by summing all the $\Delta \lambda_{\mathrm{B}}$ of the 12 FBGs and removing the mean value (Figure 2);

ii. to calibrate the smart textile in the calculation of the global volume $\left(\Delta V_{\text {global }}\right.$ Smart textile $)$ and compartmental volumes $\left(\Delta V_{\mathrm{RCp}}\right.$ Smart textile,$\Delta V_{\mathrm{RCa}}$ Smart textile, $\Delta V_{\mathrm{AB}}$ Smart textile), hereinafter called training phase.

The second trial was used to test the calibrated smart textile in the estimation of compartmental $\left(\Delta V_{\mathrm{RCp}}\right.$ Smart textile, $\Delta V_{\mathrm{RCa}}$ Smart textile, $\Delta V_{\mathrm{AB}}$ Smart textile) and global volume ( $\Delta V_{\text {global }}$ Smart textile) changes, by using the compartmental $\left(\Delta V_{\mathrm{RCp}}^{\text {MoCap }}, \quad \Delta V_{\mathrm{RCa}}{ }^{\text {MoCap }}, \Delta V_{\mathrm{AB}}^{\text {MoCap }}\right)$ and global
$\Delta V_{\text {global }}{ }^{\text {MoCap }}$ volume changes obtained by the MoCap as the reference.

\section{1 | Temporal parameters}

We used the signal obtained by summing the 12 FBG output changes (ie, $\Delta \lambda_{\mathrm{B}}$ global $)$ and the $\Delta V_{\text {global }}$ MoCap to extract all the above-mentioned temporal parameters from the smart textile and the MoCap, respectively. The steps followed are reported here below and schematically summarized in Figure 3:

i. The minimum peak at the end of the forced inspiration in the signal was identified, on both the $\Delta \lambda_{\mathrm{B}}$ global and
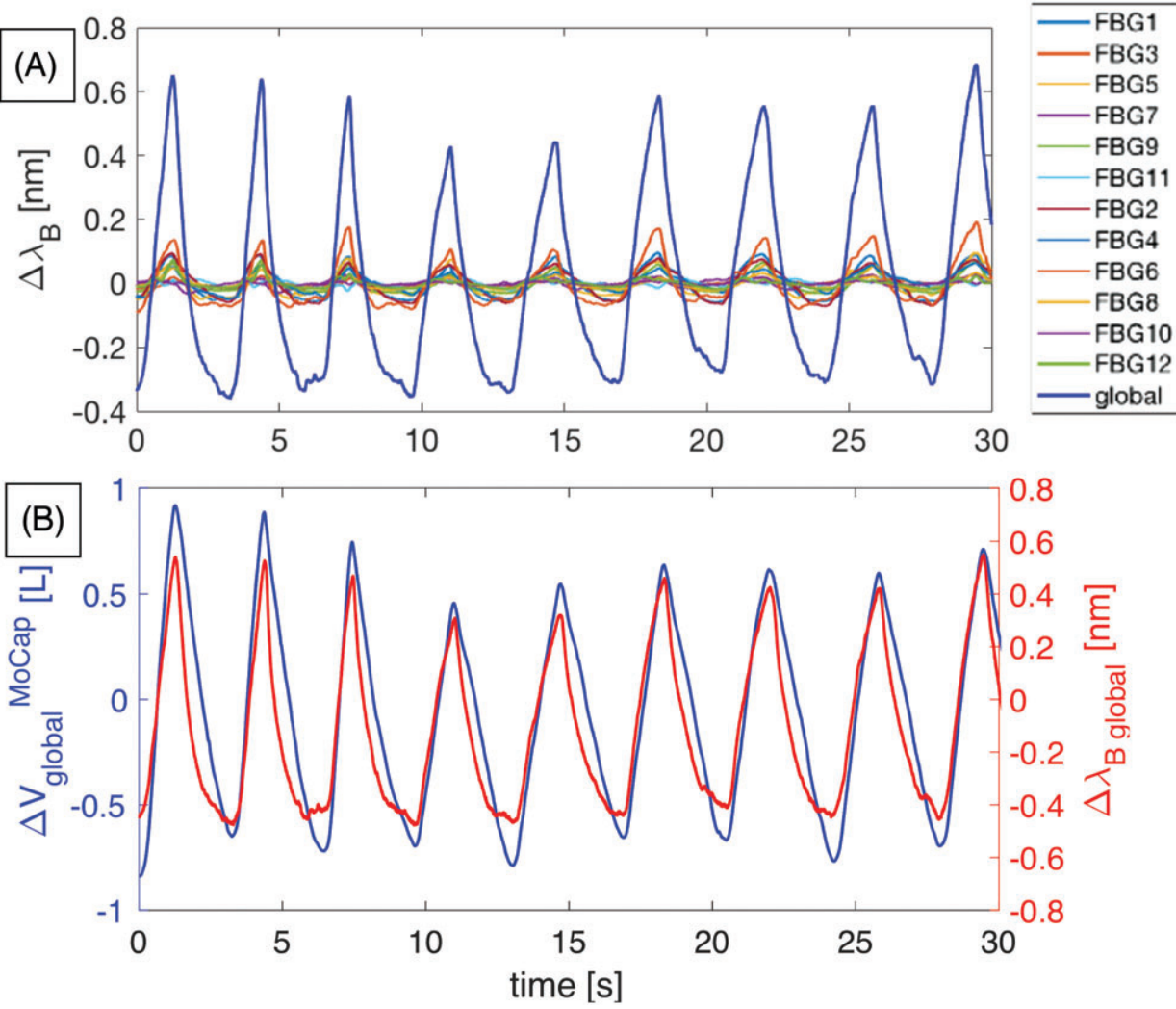

FIGURE 2 (A) Zero-mean wavelength changes $\Delta \lambda_{\mathrm{B}}$ collected by the $12 \mathrm{FBG}$ sensors and the global wavelength change ( $\Delta \lambda_{\mathrm{B} \text { global }}$ ) obtained by summing the $12 \Delta \lambda_{\mathrm{B}}$ traces. (B) Zero-mean $\Delta \lambda_{\mathrm{B} \text { global }}$ plotted on $\Delta V_{\text {global }}$ MoCap: a window of 30 seconds during quiet breathing of volunteer 1 
$\Delta V_{\text {global }}{ }^{\text {MoCap }}$ : these 2 points were used to synchronize the 2 signals.

ii. The signals were resampled at $60 \mathrm{~Hz}$ (ie, the lower sampling frequency between the 2 systems) and then their mean values were removed by the signals.

iii. Maximum and minimum peaks were identified on both the zero-mean signals by a custom-made algorithm based on both a setup time window and signal threshold (see Figure 3) [8].

Thus, each $T_{\mathrm{R}}$ value was calculated as the time elapses between 2 consecutive maximum peaks, $T_{\mathrm{I}}$, as the time interval between a maximum and its previous minimum, and $T_{\mathrm{E}}$, as the time interval between the same maximum and the following minimum. Then the breathing frequency $\left(f_{R}\right)$ - expressed in breaths per minute (breaths/min) —was calculated as the ratio between 60 and $T_{\mathrm{R}}$ values.

The comparison between all the $T_{\mathrm{R}}, f_{R}, T_{\mathrm{I}}, T_{\mathrm{E}}$ values obtained by using the 2 measurement systems was performed using 3 approaches: (i) Bland-Altman analysis was carried out to evaluate the mean of difference (MOD), as well as the limits of agreements (LOAs), calculated as the MOD \pm 1.96 the standard deviation (SD) [20]; (ii) linear regression was used to evaluate the relationships between values; (iii) a statistical test was used to investigate the hypothesis that there is no linear relationship between each parameter of interest estimated by the 2 different measuring systems ( $P$ values close to 0 correspond to a significant correlation between parameters). A significance level of 5\% was set.

Then, from $T_{\mathrm{R}}$ and $f_{R}$ values, the percentage differences between the 2 instruments $\left(\% e_{T_{R}}\right.$ and $\% e_{f_{R}}$, respectively) were calculated as in Equation (4):

$$
\begin{aligned}
& \% e_{T_{R}}=\frac{{\overline{T_{R}}}^{\text {smart textile }}-{\overline{T_{R}}}^{\text {MoCap }}}{{\overline{T_{R}}}^{\text {smart textile }}} \cdot 100 \\
& \% e_{f_{R}}=\frac{{\overline{f_{R}}}^{\text {smart textile }}-{\overline{f_{R}}}^{\text {MoCap }}}{{\overline{f_{R}}}^{\text {smart textile }}} \cdot 100
\end{aligned}
$$

being the ${\overline{T_{R}}}^{\text {smart textile }},{\overline{T_{R}}}^{\text {MoCap }},{\overline{f_{R}}}^{\text {smart textile }},{\overline{f_{R}}}^{\text {MoCap }}$ the mean value of $T_{R}$ and $f_{R}$ calculated for each volunteer using the 2 systems, respectively.

\section{2 | Breathing volumes}

We used the signal collected during the first trial by the 12 FBG output changes (ie, $\Delta \lambda_{\mathrm{B} 1} \ldots \Delta \lambda_{\mathrm{B} 12}$ ) and the $6 \mathrm{com}$ partmental volumes obtained by the MoCap to calibrate the smart textile (ie, to reconstruct the compartmental volume changes from the FBG signals).

The followed steps are reported here below:

i. By summing the signal outputs of 2 sensors that monitor the same compartment according to Figure 1 (ie, 1 and
2, 3 and 4,5 and 6,7 and 8,9 and 10,11 and 12), the 6 wavelength changes relative to the left and right sides of each compartment were obtained as shown in Figure 4 (colored rectangles);

ii. the peak at the end of the forced inspiration in the signal was identified, on both the $\Delta \lambda_{\mathrm{B}}$ global (obtained summing the 6 wavelength changes signals) and $\Delta V_{\text {global }}{ }^{\text {MoCap: }}$ since all the FBG signals are synchronized as well as all the volume changes, this peak was used as starting points of both the 6 wavelength changes signals and of the 6 compartmental volume;

iii. all the signals were resampled at $60 \mathrm{~Hz}$ (ie, the lower sampling frequency between the 2 systems) and then their mean values were removed;
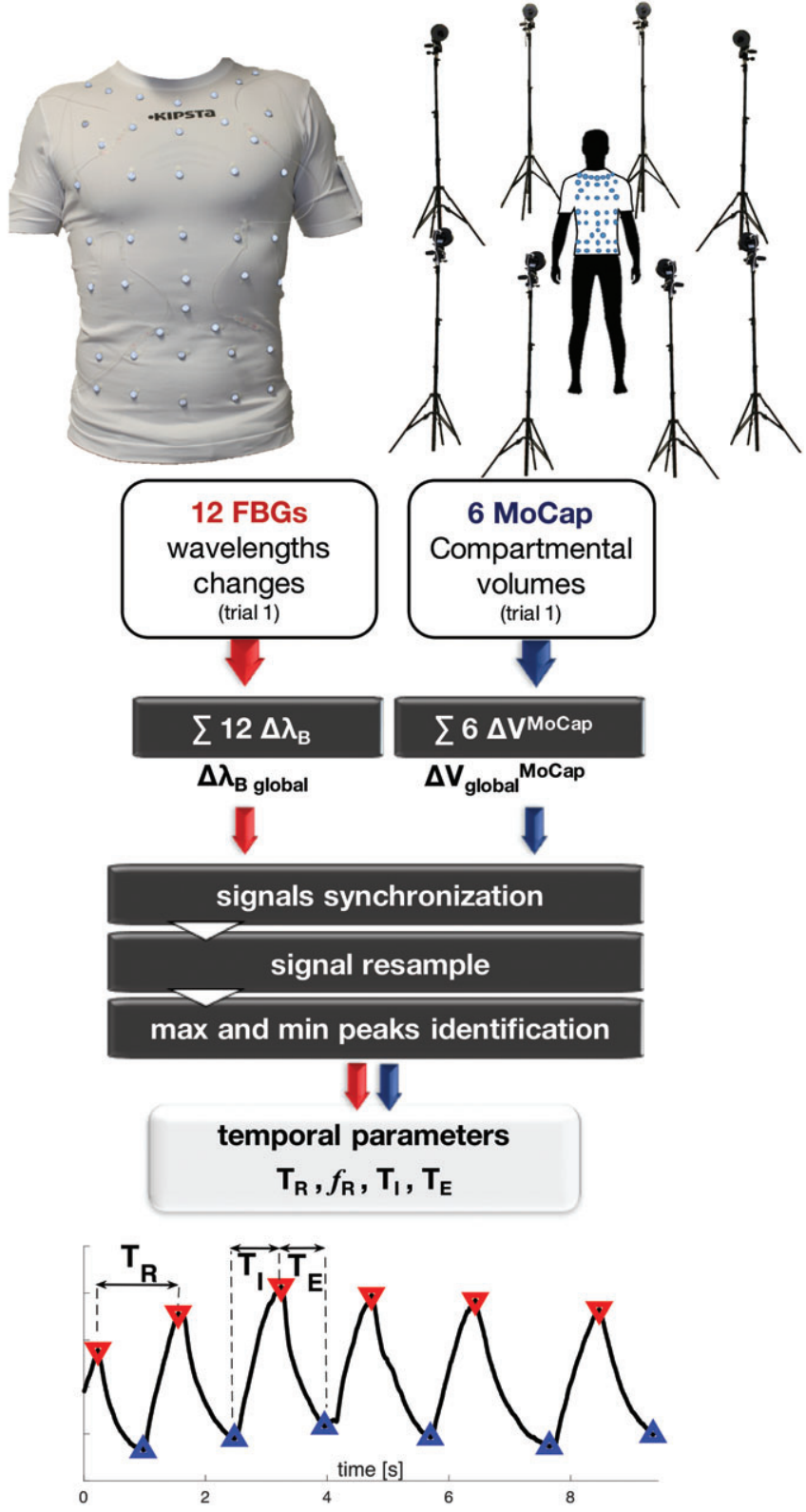

FIGURE 3 Scheme of the procedure adopted to extract temporal parameters from both the smart textile and MoCap system from data collected during trial 1 
iv. minimum peaks were identified on the 6 smart textile signals and on the 6 volume signals by the same custom-made algorithm used in the temporal parameter calculation.

In order to reconstruct the compartmental volume changes from the FBG signals, multiple linear regression was performed on each breath included between 2 consecutive minimum peaks using MATLAB function regress. The 6 smart
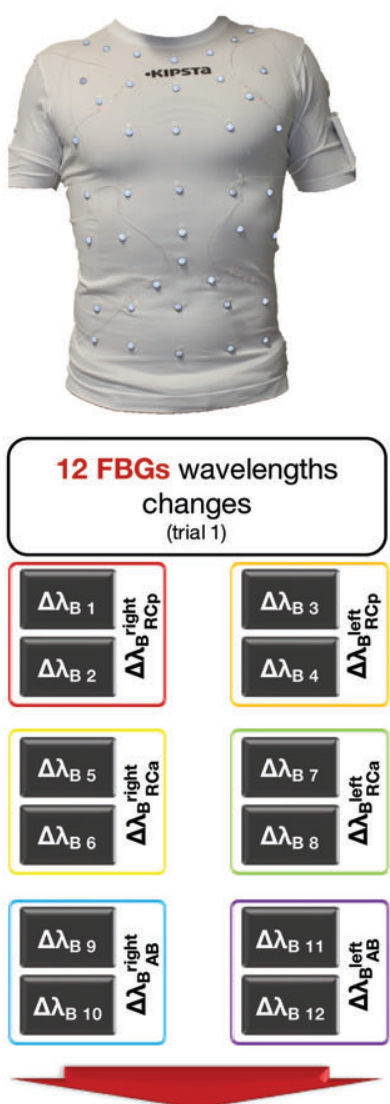

signals synchronization
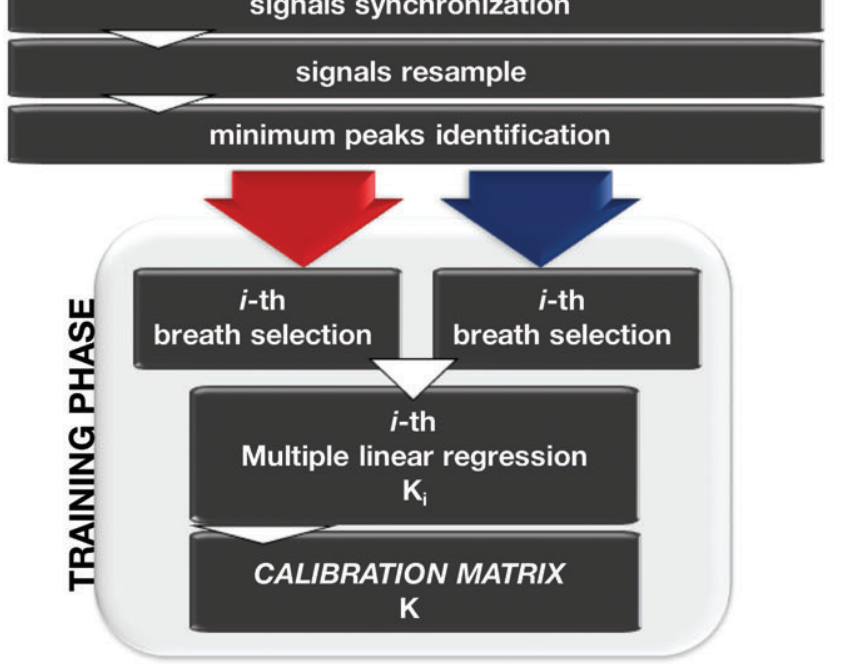

FIGURE 4 Scheme of the procedure adopted to calibrate the smart textile (training phase) in the computation of compartmental volumes textile signals and the 6 volume signals of the $j$-th breath were used in the multiple linear regression in order to obtain the $j$ th a square matrix $K_{j}(6 \times 6$ size $)$ that contains the different weights of the 6 FBG outputs in the six compartmental volume changes reconstructions as reported in Equation (5):

$$
\left(\Delta V_{i}^{\mathrm{MoCap}}\right)_{j}=K_{j} \cdot\left(\Delta \lambda_{B i}\right)_{j},
$$

where $i$ represents the 6 compartments $\left(\begin{array}{l}\text { right left } \\ R C p, r i g h t \text { left }\end{array}\right.$ ${ }_{A B}^{\text {right left }},{ }_{A B}$ ). Then the calibration matrix $K$ of each volunteer was obtained by averaging the values of $j$ square matrix $K_{j}$.

Data collected in trial 2 were used to test the smart textile in the volume calculations. The calibration matrix $K-$ obtained by the training phase-was applied to the 6 FBG signals in order to estimate the compartmental volumes $\left(\Delta V_{i}^{\mathrm{FBG}}\right)$ according to Equation (6):

$$
\Delta V_{i}^{\text {smarttextile }}=\Delta \lambda_{B i} \cdot K .
$$

Hence, from the 6 compartmental $\Delta V^{\text {smart textile }}$, left and right contributions of the same compartment were summed to obtain 3 compartmental volumes changes from the smart textile relative to the pulmonary rib cage $\left(\Delta V_{\mathrm{RCp}}\right.$ smart textile $)$, the abdominal rib cage $\left(\Delta V_{\mathrm{RCa}}\right.$ smart textile $)$, the abdomen $\left(\Delta V_{\mathrm{AB}}\right.$ smart textile $)$. The global volume changes $\left(\Delta V_{\text {global }}\right.$ smart textile) were calculated by summing the 3 compartmental volume changes.

Figure 5 shows the zero-mean global and the 3 compartmental $\Delta V^{\text {smart textile }}$ obtained after the calibration process (training phase) by data collected on a volunteer, together with the $\Delta V^{\text {MoCap }}$ contemporaneously collected.

So, the 3 compartmental and the global volume changes obtained by the calibration of the smart textile were compared to the ones saved by the MoCap. First, similarly to the trial 1 , peak at the end of the forced inspiration in the signal was identified, on both the $\Delta V_{\text {global }}$ smart textile and $\Delta V_{\text {global }}{ }^{\text {MoCap }}$, to synchronized the 4 volumes of the smart textile with the ones of the MoCap; then all the signals were resampled at $60 \mathrm{~Hz}$ and the mean removed. Then maximum and minimum peaks were identified on the $\Delta V_{\text {global }}$ smart textile and $\Delta V_{\text {global }}$ MoCap signals: tidal volume $\left(\mathrm{VT}_{i}\right)$ values were calculated as the difference between 2 consecutive minimum (end-expiratory volume) and maximum (end-inspiratory volume) points, with $i$ the quantity of the breaths selected between the 2 points.

All the $\mathrm{VT}_{i}$ values collected by the 8 volunteers per each $i$-th breath, obtained from the smart textile $\left(\mathrm{VT}_{\text {smart tex- }}\right.$ tile) were compared against the $\mathrm{VT}_{\text {MoCap }}$ obtained by the MoCap system.

Following the analysis performed on temporal parameters, the comparison between all the VT values collected by the 8 volunteers with 2 measurement systems was performed using the same 3 approaches: (i) Bland-Altman analysis; (ii) linear regression analysis; (iii) statistical test was used to investigate the hypothesis that there is linear relationship between $\mathrm{VT}_{\text {MoCap }}$ and $\mathrm{VT}_{\text {smart textile }}(P<.05)$. 


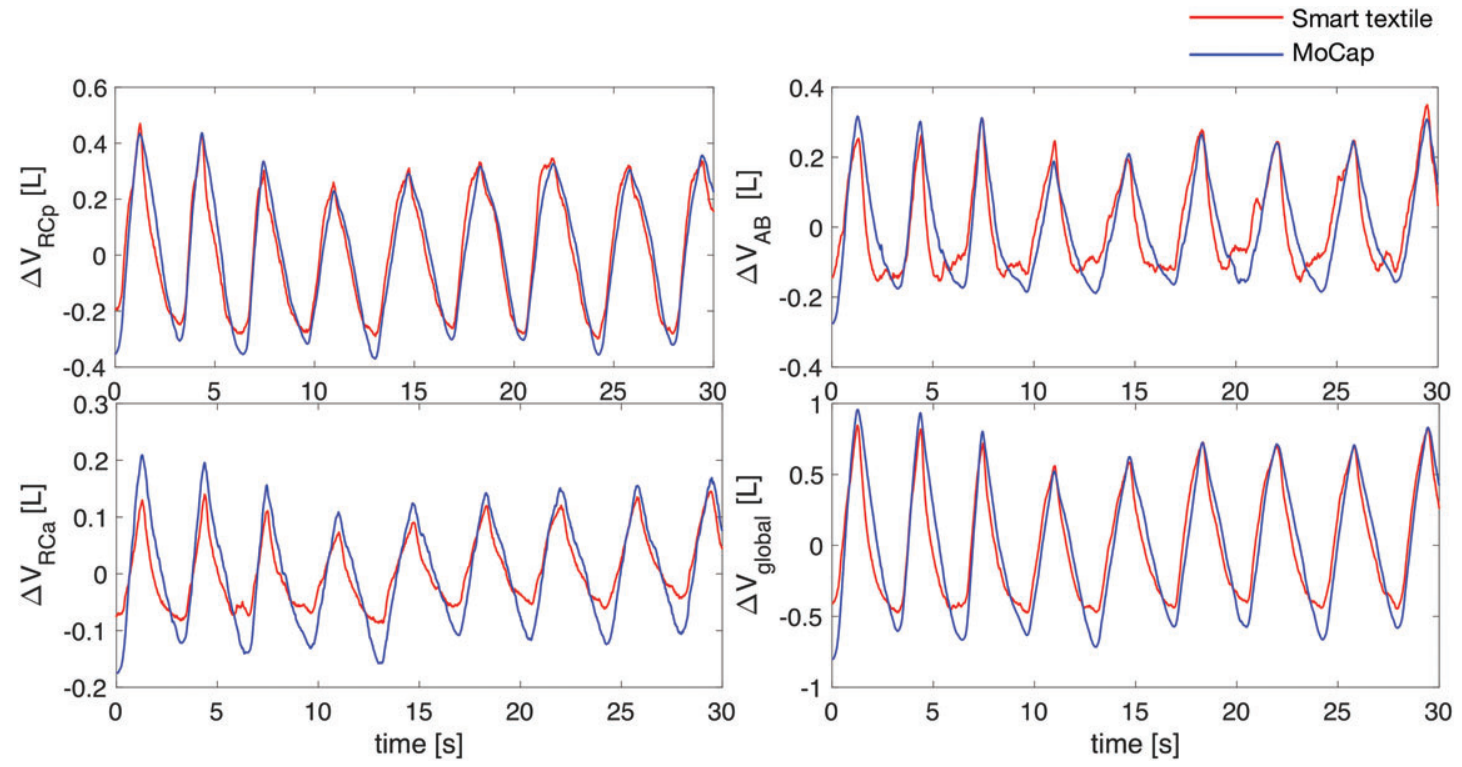

FIGURE 5 Zero-mean RCp, RCa, AB, and global volume changes recorded by the MoCap (blue lines) and obtained by the smart textile after the calibration phase (red lines)

By following the same procedure adopted to extract VT from the global signals, the compartmental VT were extracted by $\Delta \mathrm{V}_{\mathrm{RCp}}, \Delta \mathrm{V}_{\mathrm{RCa}}$ and $\Delta \mathrm{V}_{\mathrm{AB}}$ signals gathered by MoCap and calibrated smart textile. Then, average percentage contributions were calculated according to Equation (7):

$$
\left\{\begin{array}{c}
\overline{\% R C p}=\frac{\sum_{i} \% R C p^{i}}{N}=\frac{\sum_{i} \frac{V T_{R C p}^{i}}{V T^{i}} \cdot 100}{N} \\
\overline{\% R C a}=\frac{\sum_{i} \% R C a^{i}}{N}=\frac{\sum_{i} \frac{V T_{R C a}^{i}}{V T^{i}} \cdot 100}{N} \\
\overline{\% A B}=\frac{\sum_{i} \% A B^{i}}{N}=\frac{\sum_{i} \frac{V T_{A B}^{i}}{V T^{i}} \cdot 100}{N}
\end{array}\right.
$$

$i$ being the $i$-th breath considered and $N$ the number of breaths executed by the volunteer in the trial 2. Moreover, standard deviation (SD) was also calculated considering all the $N$ values of VT contributions obtained from each compartment. The SD values may be useful to evaluate the variability of the thoraco-abdominal contributions during the breathing activity. This procedure was carried out per each volunteer, separately.

To evaluate the quantitative differences between the 2 systems, raw differences $(\Delta \%)$ between $\overline{\% R C p}, \overline{\% R C a}$, $\overline{\% A B}$ were calculated as in Equation (8), by using the values gathered by the MoCap as reference:

$$
\left\{\begin{array}{l}
\Delta \%_{R C p}=\overline{\% R C p}^{\text {Smarttextile }}-\overline{\% R C p}^{\text {MoCap }} \\
\Delta \%_{R C a}=\overline{\% R C a}^{\text {Smarttextile }}-\overline{\% R C a}^{\text {MoCap }} \\
\Delta \%_{A B}=\overline{\% A B}^{\text {Smarttextile }}-\overline{\% A B}^{\text {MoCap }}
\end{array}\right.
$$

TABLE 3 Temporal parameters: summary of correlation, statistical and Bland-Altman analyses comparing the smart textile data to the reference values gathered by the MoCap for $T_{\mathrm{I}}, T_{\mathrm{E}}, T_{\mathrm{R}}, f_{\mathrm{R}}$

\begin{tabular}{llll} 
& $\boldsymbol{R}^{\mathbf{2}}$ & $\boldsymbol{P}$ value & Bias $\pm \mathbf{S D}$ \\
\hline$T_{\mathrm{I}}$ & .75 & $<.05$ & $0.15 \pm 0.31 \mathrm{~s}$ \\
$T_{\mathrm{E}}$ & .81 & $<.05$ & $-0.14 \pm 0.32 \mathrm{~s}$ \\
$T_{\mathrm{R}}$ & .97 & $<.05$ & $0.01 \pm 0.19 \mathrm{~s}$ \\
$f_{R}$ & .93 & $<.05$ & $-0.02 \pm 1.04$ breaths $/$ min
\end{tabular}

\begin{tabular}{|c|c|c|c|c|c|c|c|c|}
\hline \multirow[b]{2}{*}{ Volunteer } & \multicolumn{2}{|l|}{$T_{\mathrm{I}}(\mathbf{s})$} & \multicolumn{2}{|l|}{$T_{\mathrm{E}}(\mathrm{s})$} & \multicolumn{2}{|l|}{$\mathbf{T}_{\mathbf{R}}(\mathbf{s})$} & \multicolumn{2}{|c|}{$f_{\mathrm{R}}($ breaths/min) } \\
\hline & MoCap & Smart textile & MoCap & Smart textile & MoCap & Smart textile & МoCap & Smart textile \\
\hline 1 & $1.79 \pm 0.29$ & $1.72 \pm 0.27$ & $1.43 \pm 0.39$ & $1.49 \pm 0.33$ & $3.22 \pm 0.33$ & $3.22 \pm 0.30$ & $18.63 \pm 0.11$ & $18.63 \pm 0.10$ \\
\hline 3 & $2.60 \pm 0.27$ & $2.57 \pm 0.40$ & $2.08 \pm 0.47$ & $2.11 \pm 0.38$ & $4.68 \pm 0.43$ & $4.68 \pm 0.40$ & $12.82 \pm 0.31$ & $12.82 \pm 0.29$ \\
\hline 4 & $1.71 \pm 0.28$ & $1.68 \pm 0.23$ & $2.24 \pm 0.31$ & $2.28 \pm 0.30$ & $3.96 \pm 0.48$ & $3.96 \pm 0.46$ & $15.15 \pm 0.25$ & $15.15 \pm 0.24$ \\
\hline 7 & $2.39 \pm 0.43$ & $2.06 \pm 0.25$ & $2.83 \pm 0.46$ & $3.07 \pm 0.34$ & $5.22 \pm 0.64$ & $5.13 \pm 0.37$ & $11.49 \pm 0.58$ & $11.70 \pm 0.32$ \\
\hline 8 & $1.63 \pm 0.39$ & $1.22 \pm 0.14$ & $2.00 \pm 0.15$ & $2.41 \pm 0.26$ & $3.63 \pm 0.31$ & $3.63 \pm 0.33$ & $16.53 \pm 0.14$ & $16.53 \pm 0.15$ \\
\hline
\end{tabular}

TABLE 2 Duration of the inspiratory phase $\left(T_{\mathrm{I}}\right)$, expiratory phase $\left(T_{\mathrm{E}}\right)$, breathing duration $\left(T_{\mathrm{R}}\right)$ and breathing frequency $\left(f_{R}\right)$

Mean and standard deviation (SD) values are reported for both the smart textile and the MoCap system, for each volunteer. 

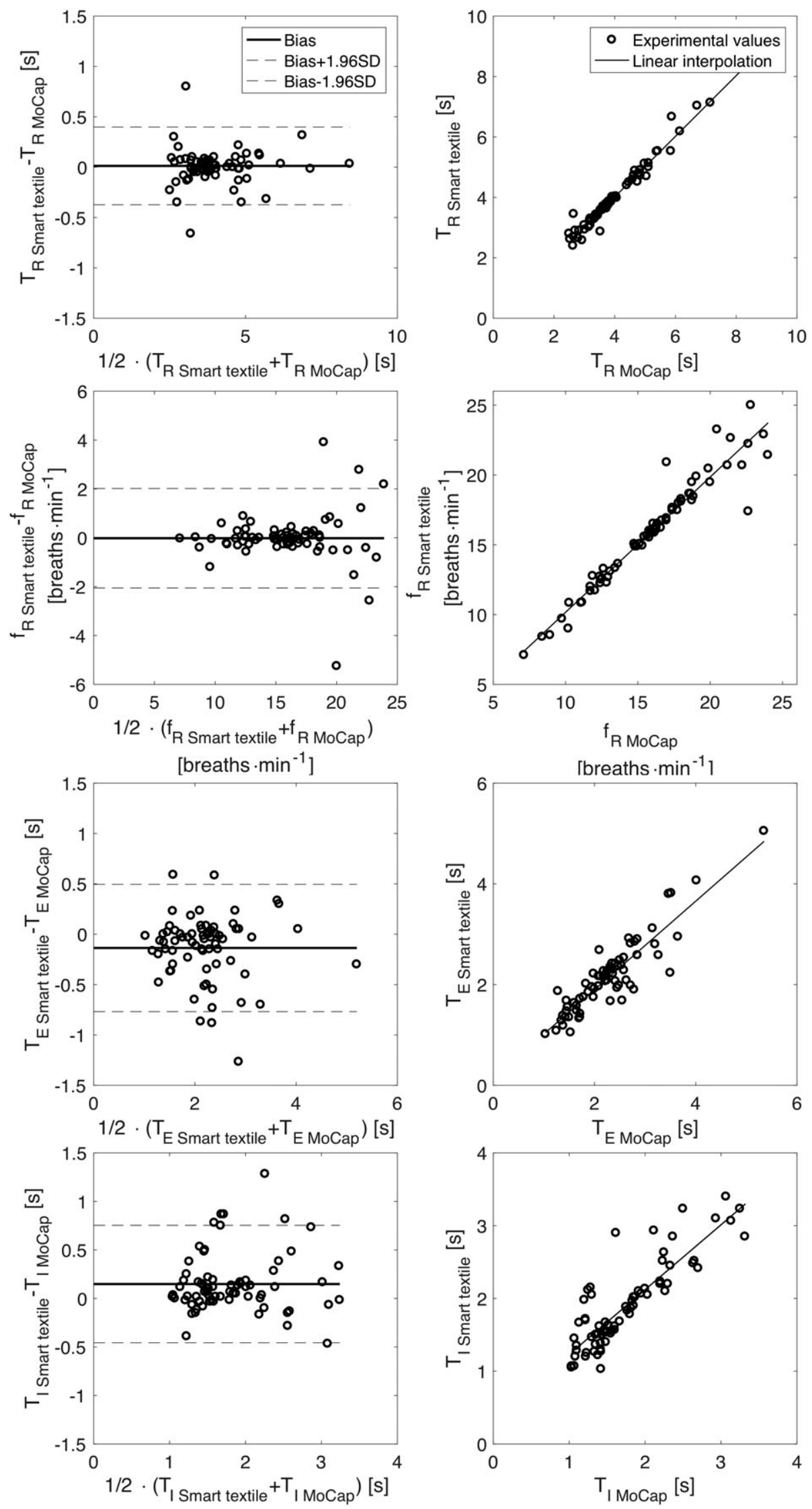

FIGURE $6 T_{\mathrm{R}}, f_{R}, T_{\mathrm{E}}$ and $T_{\mathrm{I}}$ values calculated by the smart textile and MoCap used as reference. For each parameter, the linear relationship between the values gathered by smart textile and the MoCap is reported. Bland and Altman's analysis plots comparing the values $(n=71)$ of $T_{\mathrm{R}}, f_{\mathrm{R}}, T_{\mathrm{E}}$ and $T_{\mathrm{I}}$ between the 2 systems are also shown. The thick continuous line shows the bias, whereas the dotted lines show the upper and lower limit of agreements (LOAs) 

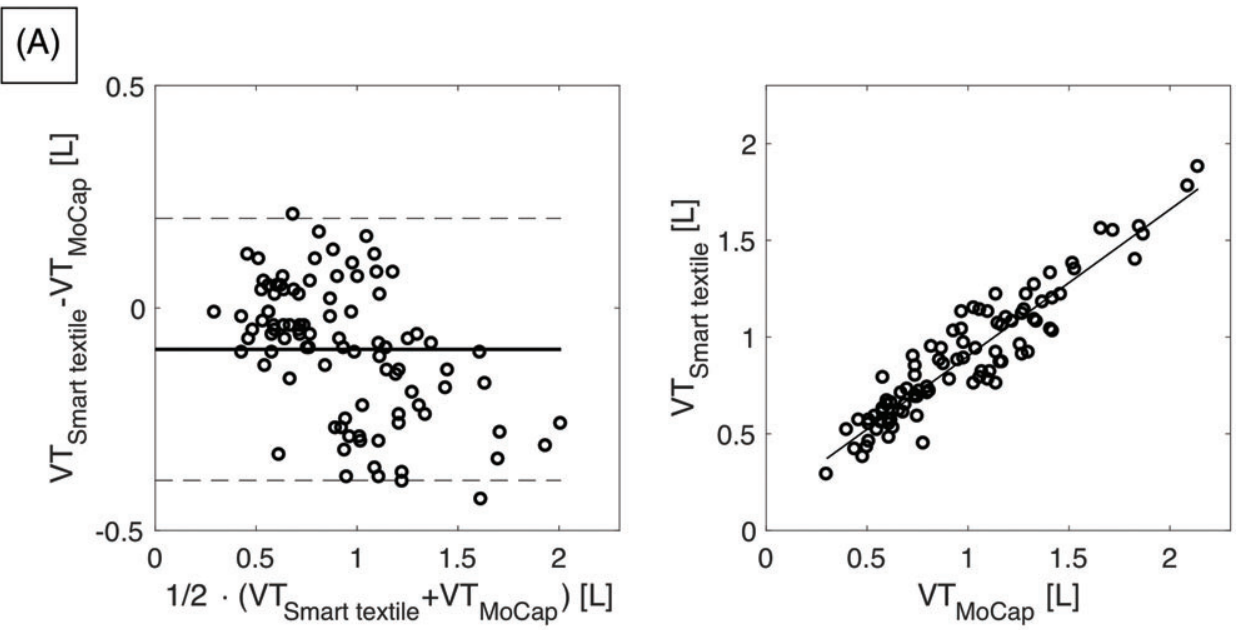

FIGURE 7 (A) VT values calculated by the smart textile compared against the ones gathered by the MoCap: Bland Altman and linear relationship graphs. In the Bland Altman, the thick continuous line shows the bias, whereas the dotted lines the limits of agreement (LOAs) (B) VT mean and SD values (gray error bars) for each volunteer: In blue, the values collected by the MoCap, red bars the ones collected by the smart textile

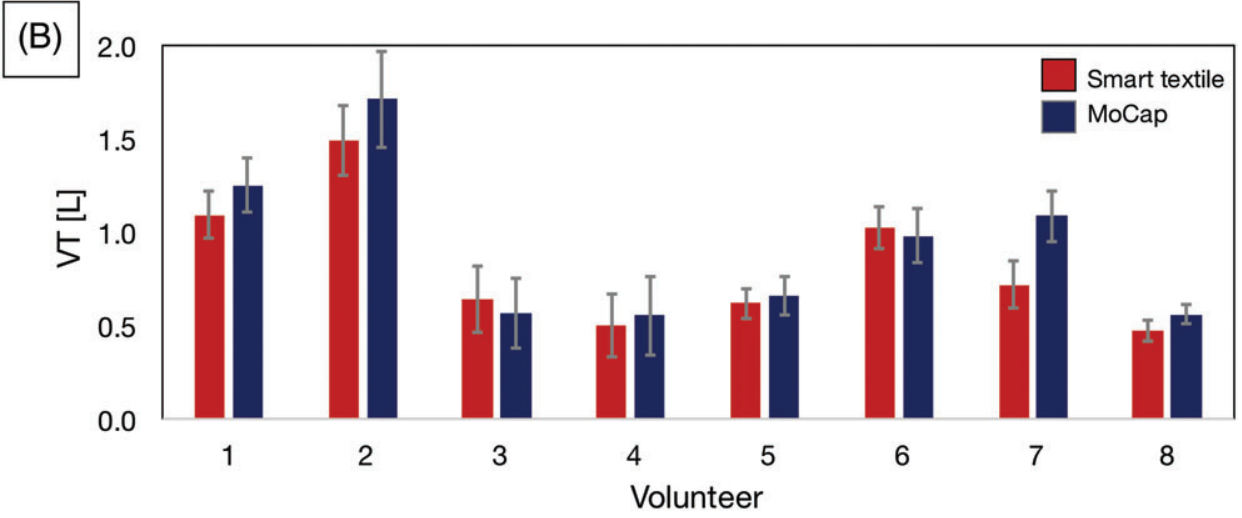

In order to investigate the statistical differences between the smart textile and the reference instrument in the percentage contributions, statistical test was applied to investigate the hypothesis that there is linear relationship $(P<.05)$.

\section{I RESULTS}

\section{1 | Temporal parameters}

Table 2 shows the mean and the SD values of each temporal parameter $\left(T_{\mathrm{I}}, T_{\mathrm{E}}, T_{\mathrm{R}}, f_{\mathrm{R}}\right)$ obtained by the 2 systems for each volunteer. The SD was calculated by considering all the breaths in each signal collected during the trial. Table 3 shows the results of the Bland Altman analysis (expressed as $\mathrm{MOD} \pm \mathrm{SD}$ ), of the linear regression $\left(R^{2}\right)$ and of the statistical test ( $P$ value).

Both $T_{\mathrm{R}}$ and $f_{\mathrm{R}}$ estimated by the smart textile showed a good agreement with the reference ones. This is testified by (i) the results of the linear regression (Figure 6): high $R^{2}$ values (.97 and .93, respectively) and slope of the best fitting line close to 1 (.99 and .99 , respectively); by (ii) the results of the Bland Altman analysis (Figure 6): the low value of the bias ( 0.01 seconds for $T_{\mathrm{R}}$ and -0.02 breaths/ min for $\mathrm{f}_{\mathrm{R}}$ ); by (iii) the low value of the maximum $\% e_{T_{R}}$ and $\% e_{f_{R}}$ : they were -0.09 seconds $(1.72 \%)$ and -0.20 breaths $/ \min (-1.75 \%)$, while the average values were $0.27 \%$ for both. Finally, a linear relationship between the 2 parameter estimated by the 2 systems was statistically significant $(P<.05)$, as expected.

\section{2 | Breathing volumes}

Performance of the smart textile in the calculation of global volume changes was evaluated by comparing the breath-bybreath tidal volume extracted by the $\Delta V_{\text {global }}^{\mathrm{FBG}}\left(\mathrm{VT}_{\mathrm{Smart}}\right.$ textile) against the tidal volumes extracted by $\Delta V_{\text {MoCap }}^{\text {global }}$ $\left(\mathrm{VT}_{\text {reference }}\right)$, used as reference values.

Figure 7A shows the BA graph (with MOD and LOAs) and the linear regression used to evaluate the relationships between VT values.

The Bland-Altman analysis showed a mean bias value of $-0.09 \pm 0.15 \mathrm{~L}$ suggesting that the smart textile slightly underestimates VT, pointing out the good agreement between the 2 systems $\left(\mathrm{R}^{2}=.90, P<.05\right)$.

The mean values of the $\mathrm{VT}_{\text {Smart textile }}$ and $\mathrm{VT}_{\text {reference }}$ values calculated per each volunteer are shown in Figure 7B, together with the SD calculated by all the VT values of the same volunteer. Linear relationship was found between the mean values of VT collected by the 2 instruments $(P<.05)$.

Regarding the thoraco-abdominal patterns, Figure 8 shows the compartmental percentage contributions (ie, \% 

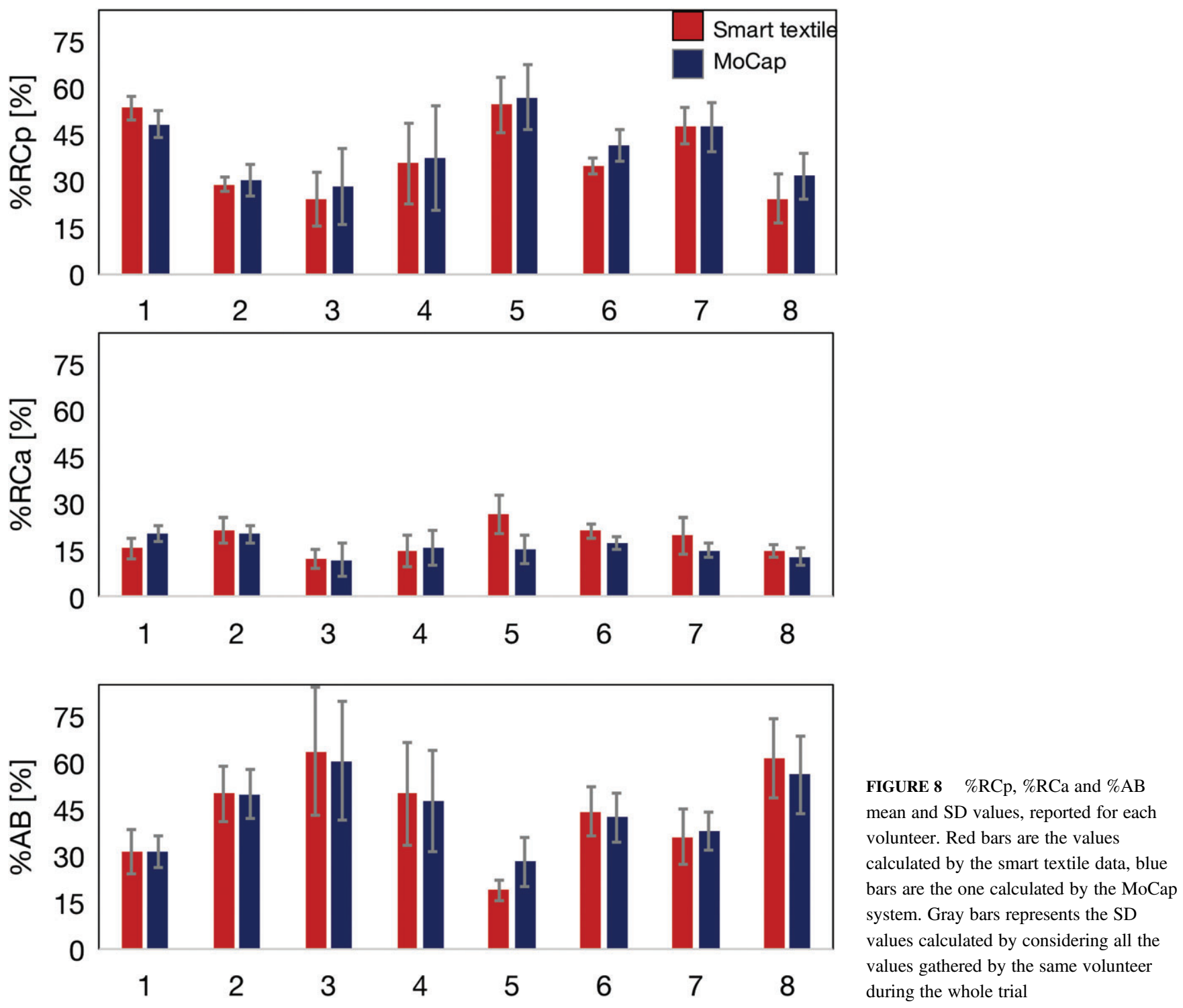

$\mathrm{RCp}, \% \mathrm{RCa}, \% \mathrm{AB})$ calculated by the 2 instruments, per each volunteer.

The raw differences $\left(\Delta \%_{\mathrm{RCp}}, \Delta \%_{\mathrm{RCa}}, \Delta \%_{\mathrm{AB}}\right)$ were calculated. The average values were $-2.2 \%$ for the $\Delta \%_{\mathrm{RCp}}$, $2.2 \%$ for $\Delta \%_{\mathrm{RCa}}, 0.2 \%$ for $\Delta \%_{\mathrm{AB}}$.

By considering all compartmental percentage values for all the volunteers enrolled, we found linear relationship between values gathered by the 2 systems $(P<.05$ for $\%$ $\mathrm{RCp}, \% \mathrm{RCa}$ and \%AB).

\section{6 | DISCUSSION AND CONCLUSIONS}

Breathing frequency and temporal patterns changes can highlight serious adverse respiratory events or early signs of the respiratory diseases processes [1]. Moreover, the observation of breathing may play a fundamental role in many other applications, including sport studies, quarantine, and security examinations. Recent technological advances in wearable sensors and wireless communication make it possible to design non-invasive, intelligent, and lightweight healthcare monitoring systems for the respiratory frequency monitoring [2]. Although respiratory parameters are of paramount importance to monitor the rapid changes of the physiological state of the subject, the breath-by-breath monitoring of both breathing frequency and tidal volume is not common in commercial devices and in validation studies. Additionally, despite the analysis of thoraco-abdominal contributions is gaining interests in both clinical and research scenarios [15], few number of wearable solutions have been developed to collect the upper and lower thorax temporal pattern [21].

In this paper, we experimentally evaluate a wearable smart textile integrating 12 FBGs as sensing elements, for the non-intrusive breath-by-breath monitoring of both temporal parameters (eg, duration of each breath, respiratory rate, inspiratory and expiratory phase durations) and breathby-breath respiratory volume changes (of the whole chest wall and ist compartments). This smart textile has been designed to embed 12 FBGs to optimize the sensitivity of the system in the monitoring of breath-by-breath respiratory temporal parameters. It has been previously tested on a 
small population by using a flowmeter as reference instrument [16]. In the present work, a MoCap system with specific software for breathing analysis [22] has been used to collect the reference values of temporal parameters and the respiratory volumes. The working principle of both the MoCap system and the proposed smart textile is based on the measurement of chest wall movements which occur during the breathing activity [10].

The proposed textile shows biases of $0.01 \pm 0.19 \mathrm{sec}-$ onds and $-0.02 \pm 1.04$ breaths/min in the breath-by-breath calculation of respiratory period and breathing rate, respectively, and a strong correlation with data collected by MoCap $\left(R^{2}=.97\right.$ and .93 , respectively).

These results, togheter with biases found in the breathby-breath inspiratory and expiratory phase durations values (up to 0.15 seconds), are similar to values reported in previous studies adopting fiber optic-based garments for the breathing rate monitoring [13, 14, 19, 23, 24]. In addition, the $f_{\mathrm{R}}$ estimation show that results herein reported are similar to those shown in a previous paper focused on the smart textile under test. These results highlighted that after 1 year of use the performance of the proposed garment did not show any drift.

Regarding the respiratory volumes monitoring, the proposed smart textile demonstrated an overall slight underestimation of breath-by-breath VT values with a bias of $-0.09 \pm 0.15 \mathrm{~L}$ and strong correlation with reference measurements $\left(R^{2}=.90, P<.05\right)$.

When compartmental volume contributions collected by the smart textile were compared to the MoCap values, a maximum overall difference of $+2.2 \%$ in the RCp contribution was found, that was compensated by a difference of $-2.2 \%$ in the RCa compartment, similarly to results obtained in Reference [14]. Significant linear relationship between smart textile and MoCap percentage contribution values was found $(P<.05)$. It means that the proposed smart textile can be potentially used to identify abnormal breathing pattern and disorders within the respiratory system and help refine the differential diagnosis in clinical practise [15] by the analysis of thoraco-abdominal patterns, and it should be used to can indirectly measure the muscular involvement, and the coordination of the breathing during the effort in sport science [25].

Since only healthy male participants have been recruited to experimentally test the garment during quiet breathing, further experiments will be required to investigate the usability of the system in the monitoring of breathing parameters and thoraco-abdominal patterns during other physical activity differently from the quiet breathing (ie, walking, running, cycling), overcoming the limitations of MoCap technologies nowadays adopted in research scenario [15]. Additionally, female volunteers will be included in next experimental tests. Nevertheless, the authors recognize that future work has to be done in order to evaluate the accuracy of volume measurements between tests on the same volunteer by adopting the same calibration matrix; this investigation will allow figuring out the intra-subject variability within different trials [26].

In conclusion, this paper demonstrates the possibility to monitor the breathing temporal parameters, the breath-bybreath tidal volume, the thoraco-abdominal motion patterns and their variations during the breathing activity on healthy volunteers by wearing a FBG-based smart textile. These features open up the further usage of the proposed textile in wide applications in ambulatory and sport science settings.

\section{ACKNOWLEDGMENTS}

The authors thank Dott. Michelangelo Morrone and Prof. Silvia Sterzi (Physical Medicine and Rehabilitation Unit, Università Campus Bio-Medico di Roma) for their helpful contribution in MoCap data collection. Additionally, they gratefully acknowledge Dott. Andrea Polimadei (ENEA Frascati) for his help in the optical fiber manufacturing.

\section{AUTHOR BIOGRAPHIES}

Please see Supporting Information online.

\section{REFERENCES}

[1] G. Yuan, N. A. Drost, R. A. McIvor, Clin. Rev. 2013, 10(1), 23.

[2] A. Aliverti, Breathe 2017, 13(2), e27.

[3] S. Majumder, T. Mondal, M. Deen, Sensors 2017, 17(1), 130.

[4] C. Massaroni, P. Saccomandi, E. Schena, J. Funct. Biomater 2015 6(2), 204.

[5] B. M. Quandt, L. J. Scherer, L. F. Boesel, M. Wolf, G.-L. Bona, R. M. Rossi, Adv. Healthc Mater. 2015, 4(3), 330.

[6] L. Dziuda, F. W. Skibniewski, M. Krej, J. Lewandowski, IEEE Trans. Biomed. Eng. 2012, 59(7), 1934.

[7] K. Chethana, A. S. Guru Prasad, S. N. Omkar, S. Asokan, J. Biophotonics 2017, 10(2), 278

[8] M. Ciocchetti, C. Massaroni, P. Saccomandi, M. A. Caponero, A. Polimadei, D. Formica, E. Schena, Biosensors 2015, 5(3), 602.

[9] C. Massaroni, P. Saccomandi, D. Formica, D. Lo Presti, M. A. Caponero, G. Di Tomaso, F. Giurazza, M. Muto, E. S. Schena, IEEE Sens. J. 2016 16(22), 8103

[10] K. Konno, J. Mead, J. Appl. Physiol. 1967, 22(3), 407.

[11] S. J. Cala, C. M. Kenyon, G. Ferrigno, P. Carnevali, A. Aliverti, A. Pedotti, P. T. Macklem, D. F. Rochester, J. Appl. Physiol. 1996, 81, 2680.

[12] R. L. Dellacà, A. Aliverti, P. Pelosi, E. Carlesso, D. Chiumello, A. Pedotti, L. Gattinoni, Crit. Care Med. 2001, 1807, 29(9), 1807.

[13] V. F. Parreira, D. S. R. Vieira, M. A. C. Myrrha, I. M. B. S. Pessoa, S. M. Lage, R. R. Britto, Rev. Bras. Fisioter. 2012, 16(6), 439.

[14] I. Vogiatzis, O. Georgiadou, S. Golemati, A. Aliverti, E. Kosmas, E. Kastanakis, N. Geladas, A. Koutsoukou, S. Nanas, S. Zakynthinos, C. Roussos, Thorax 2005, 60(9), 723

[15] C. Massaroni, E. Carraro, A. Vianello, S. Miccinilli, M. Morrone, I. K. Levai, E. Schena, P. Saccomandi, S. Sterzi, J. W. Dickinson, S. Winter, S. Silvestri, Respiration 2017, 93(5), 339.

[16] D. Lo Presti, C. Massaroni, D. Formica, P. Saccomandi, F. Giurazza M. A. Caponero, E. Schena, IEEE Sens. J. 2017, 17(18), 6037.

[17] D. Krohn, M. Trevor, M. Alexis, Fiber Optic Sensors Fundamentals and Applications, SPIE, Bellingham, Washington, USA : SPIE Press, 2014, p. 151.

[18] BTS Bioengineering. in Optoelectronic Plethysmography Compedium Marker Setup: A Handbook about Marker Positioning on Subjects in 
Standing and Supine Positions, BTS Bioengineering CORP.: Milan 2011, p. 1.

[19] C. Massaroni, G. Senesi, E. Schena, S. Silvestri, Comput. Methods Biomech. Biomed. Engin. 2017, 20(16), 1678.

[20] D. Giavarina, Biochem. Med. 2015, 25(2), 141.

[21] G. K. Wolf, J. H. Arnold, Crit. Care Med. 2005, 33(3), S163.

[22] C. Massaroni, E. Cassetta, S. Silvestri, J. Appl. Biomech. 2017, 33(5), 361.

[23] Ł. Dziuda, F. W. Skibniewski, M. Krej, P. M. Baran, J. Biomed. Opt. 2013, 18(5), 57006.

[24] Y. Retory, P. Niedzialkowski, C. De Picciotto, M. Bonay, M. Petitjean, PLoS One 2016, 11(3), e0151983.

[25] A. P. Silvatti, K. J. Sarro, P. Cerveri, G. Baroni, R. M. L. Barros, J. Sports Sci. 2012, 30(14), 1551.
[26] R. Dumond, S. Gastinger, H. A. Rahman, A. Le Faucheur, P. Quinton, H. Kang, J. Prioux, Eur. J. Appl. Physiol. 2017, 117(8), 1533.

How to cite this article: Massaroni $\mathrm{C}$, Venanzi C, Silvatti AP, et al. Smart textile for respiratory monitoring and thoraco-abdominal motion pattern evaluation. J. Biophotonics. 2018; 201700263. https://doi. org/10.1002/jbio.201700263 